\title{
Diagnostic interval for non-screening patients undergoing mammography during the COVID-19 pandemic
}

\author{
Priscila Crivellaro $^{1,2} \cdot$ Monica Tafur ${ }^{1,2} \cdot$ Ralph George $^{1,3} \cdot$ Derek Muradali $^{1,2}$ (D) \\ Received: 12 February 2021 / Revised: 7 May 2021 / Accepted: 1 June 2021 / Published online: 18 June 2021 \\ (C) European Society of Radiology 2021
}

\begin{abstract}
Objective During the COVID-19 pandemic, there was a temporary cessation of mammography screening. However, in some facilities, diagnostic breast imaging services continued for patients with a high clinical suspicion of breast cancer. The objective of this study was to evaluate changes in the diagnostic interval (DI) of non-screening patients presenting for diagnostic mammography during the first wave of the COVID-19 pandemic.

Methods Retrospective chart review was performed on patients presenting for non-screening diagnostic mammography from April 1 to June 30, 2020 (pandemic group) and April 1 to June 30, 2019 (pre-pandemic group). Age, reason for referral, number and type of imaging studies/biopsies necessary for a final diagnosis were recorded. Diagnostic interval (DI) was defined as the number of days from the date of the diagnostic mammogram to the date of the final diagnosis.

Results Compared to the pre-pandemic group $(n=64)$, the pandemic group $(n=77)$ showed a reduction in DI of the entire cohort (pandemic: 1 day; pre-pandemic: 15 days, $p<0.0001$ ) for patients not requiring tissue sampling (pandemic: 1 day; pre-pandemic: 11 days, $. p<0.0001$ ) and those requiring tissue sampling with benign pathology (pandemic 9 days; pre-pandemic, 33 days, $p=$ 0.0002). A higher percentage of patients in the pandemic group had their assessment completed during the initial visit (pandemic: $50.6 \%$; pre-pandemic: $23.4 \%, p=0.0009$ ).

Conclusion During the first wave of the COVID-19 pandemic, the DI for patients with non-screening-related diagnostic mammography was significantly shorter, with a higher percentage of patients completing their assessments on the initial visit, compared to one year prior.

Key Points

- Despite reductions in manpower and clinical services, during pandemic times, it is possible to maintain a diagnostic breast imaging service for women at high clinical suspicion for breast cancer.

- During pandemic times, breast imaging departments should consider restructuring to a Rapid Diagnostic Unit model with a navigation team that follows patients through the assessment process to a final diagnosis.

- Departmental restructuring and patient navigation during pandemic times could either maintain or shorten the diagnostic interval for patients presenting for diagnostic mammography.
\end{abstract}

Keywords Breast $\cdot$ Mammogram $\cdot$ COVID-19 $\cdot$ Diagnostic interval

Derek Muradali

Derek.Muradali@unityhealth.to

1 Department of Medical Imaging, St. Michael's Hospital, 30 Bond Street, Toronto, Ontario M5B 1W8, Canada

2 Department of Medical Imaging, University of Toronto, 263 McCaul Street, 4th Floor, Toronto, Ontario M5T 1W7, Canada

3 Department of Surgery, University of Toronto, 149 College Street, 5th floor, Toronto, Ontario M5T 1P5, Canada

$\begin{array}{ll}\text { Abbreviations } \\ \text { ADH } & \text { Atypical ductal hyperplasia } \\ \text { BI-RADS } & \text { Breast Imaging-Reporting and Data System } \\ \text { COVID-19 } & \text { Coronavirus disease 2019 } \\ \text { DCIS } & \text { Ductal carcinoma in situ } \\ \text { DI } & \text { Diagnostic interval } \\ \text { FNA } & \text { Fine-needle aspiration } \\ \text { IDC } & \text { Invasive ductal carcinoma } \\ \text { MRI } & \text { Magnetic resonance } \\ \text { RDU } & \text { Rapid diagnostic unit }\end{array}$




\section{Introduction}

During the first wave of the COVID-19 pandemic, a temporary cessation of mammography screening was recommended by organised screening programmes in various countries, including the UK, Australia, Netherlands and Canada [1-4]. This was concordant with decisions made throughout the healthcare system where a slowdown of non-urgent services was encouraged due to the risk of screening participants and healthcare workers becoming infected. Furthermore, deferring cancer screening to a later time enabled facilities to preserve resources for possible frontline interactions and permitted their staff to self-isolate away from the facility if needed $[1$, $5,6]$.

The referral processes and diagnostic pathways were altered during the pandemic due to institutional-wide restructuring, decreases in manpower and reduction of services [7]. In order to maintain a referral service for patients with a high clinical suspicion for breast cancer, our breast imaging department maintained a diagnostic service for patients with symptomatic presentations and those with a history of breast cancer.

Prior to the pandemic, referrals for diagnostic mammography were accepted based on their arrival time, and patients were navigated through the assessment process by either a departmental nurse or the administrative staff of referring clinicians. The wait-time for follow-up imaging tests and biopsies was determined by booking availability within the department, and the time to access the report by the referring clinician.

At the onset of the pandemic, the breast imaging department was restructured to a Rapid Diagnostic Unit (RDU) model with the objective of completing all diagnostic tests on a single or minimal number of visits. Referrals were triaged by the chief breast surgeon to ensure that all patients were of high clinical suspicion for breast cancer. The navigation team was expanded to include mammography technologists, radiologists, surgeons, secretaries and clerical staff. Patients were offered to complete all diagnostic tests on the initial visit, and if declined, follow-up tests were expedited at the convenience of the patient. All patients who underwent image-guided biopsies were referred to a breast surgeon, who contacted the patient by phone within $24 \mathrm{~h}$ of the biopsy result to discuss further management options. The restructuring did not affect the other areas of the radiology department as the breast section personnel worked there almost exclusively. However, it was unclear whether this process, coupled with the restriction of hospital services, would result in a delay in diagnosis of patients presenting for diagnostic mammography.

Delays in diagnostic times for breast cancer have been associated with substantial patient anxiety, the elevated likelihood of lymph node metastasis and increased tumour size, resulting in a negative prognosis [8]. Treatment delays can result in higher rates of local recurrence and decreased disease-free and cause-specific survival among women with early-stage breast cancer [9-11]. Based on the rapidity of the COVID-19 pandemic, with its effect on the exacerbation of anxiety in populations [12], preservation of the time to diagnosis for breast cancer to pre-pandemic levels would be desirable for the mental health and prognosis of those with breast cancer.

The objective of this study was to evaluate changes in the diagnostic interval (DI) of non-screening patients presenting to our breast imaging department, undergoing diagnostic mammography, during the first wave of the COVID-19 pandemic, compared to one year prior.

\section{Methods}

\section{Study population and data collection}

At our department, after April 1, 2020, there was a reduction of all services as a consequence of COVID-19 precautions. Although breast screening was officially halted, screening mammography continued for patients who could not be contacted with a cancellation or insisted on screening during the pandemic.

Diagnostic mammography, defined as a mammogram performed on a non-screening patient with breast-related symptoms or surveillance mammography (performed for routine monitoring of women with a history of breast cancer), continued for those patients presenting with a high clinical suspicion for breast cancer. Patients were confirmed to be at a high clinical suspicion for breast cancer after review of the referral by the chief breast surgeon and accepted for entry to the RDU on the presumption that they were COVID-19 negative based on interviews using hospital approved questioners. The imaging workup was supervised by one of two breast-fellowship trained radiologists with 31 and 26 years of post-fellowship experience.

A retrospective chart review was performed on all patients presenting for diagnostic mammography from April 1, 2020, to June 30, 2020 (representing the pandemic group) and from April 1, 2019, to June 30, 2019 (representing the pre-pandemic group). The study was approved by the Hospital Academic Health Sciences Network Research Ethics Board. Informed consent was waived.

For each patient, the following were recorded: age, gender, the reason for referral (symptom/sign, surveillance), number and type of studies (mammographic views, ultrasound, MRI) and procedures (ultrasound/stereotactic/MRI-guided core biopsy, fine-needle aspiration biopsy (FNA), surgical biopsy) necessary to determine the final diagnosis. Assessments were also classified with regard to the visit number (initial, second, third, etc.).

Imaging studies were reported using the American College of Radiology Breast Imaging-Reporting and Data System (BI- 
Table 1 American College of Radiology Breast Imaging-Reporting and Data System (BI-RADS) assessment categories. Each imaging report is assigned a category between 0 and 6 , the definitions of which are demonstrated in this table

\begin{tabular}{ll}
\hline BI-RADS & Description \\
\hline Category 0 & Incomplete - need additional imaging evaluation (and/or prior mammograms for comparison) \\
Category 1 & Negative \\
Category 2 & Benign \\
Category 3 & Probably benign \\
Category 4 & Suspicious \\
Category 5 & Highly suggestive of malignancy \\
Category 6 & Known biopsy-proven malignancy \\
\hline
\end{tabular}

RADS) lexicon (Table 1) [13]. A final benign diagnosis was established for those with a BI-RADS 1, 2 or 3 classification after all imaging studies and biopsies were completed. A final malignant diagnosis was established for those individuals with a malignant tissue biopsy.

\section{Diagnostic interval}

Diagnostic interval (DI) was defined as the number of days from the diagnostic mammogram at our institution to the final diagnosis $[14,15]$. In cases where tissue sampling was needed for diagnosis, the date of biopsy was considered as the date of the final diagnosis.

\section{Statistical analysis}

Statistical analysis was performed using Stata 14.2 software (StataCorp 2017). Descriptive statistics of the study population demographics, imaging studies and pathology results were calculated. Categorical variables were described as percentages, and continuous variables were summarised, calculating medians and ranges. Percentages were compared between the pandemic and the pre-pandemic groups using the equality of proportions test. The Wilcoxon rank-sum test was used to compare the DI between patients in both groups. A $p$ value $<0.05$ was considered statistically significant.

\section{Results}

\section{Diagnostic mammography during the COVID-19 pan- demic: April 2020-June 2020}

A total of 576 patients underwent either screening or diagnostic mammography between April 1, 2020, and June 30, 2020. Seventy-seven patients (cisgender female 74 , cisgender male 1 , transgender female 2), with a median age of 50 years (range 25-80 years), underwent diagnostic mammography for either breast-related symptoms (62) or an abnormal surveillance mammogram (15). Breast symptoms/signs included palpable lump (46), nipple discharge (3), nipple retraction (2) and pain (21), with some patients presenting with more than one symptom.

At the initial visit, 77 patients underwent a total of 133 additional imaging studies and 12 image-guided biopsies. Thirty-eight patients returned for a second visit with a total of 34 imaging studies and 18 image-guided biopsies. Eight patients returned for a third visit with a total 3 imaging studies and 8 biopsies. One patient returned for a fourth visit for MRI-guided biopsy (Table 2).

\section{Diagnostic interval}

$50.6 \%$ of patients completed their assessment on the initial visit (as exemplified in Fig. 1), 39.0\% on the second visit, $9.1 \%$ on the third visit and $1.3 \%$ on the fourth visit (Table 3). A total of 34 patients underwent image-guided biopsies, $16(47.1 \%)$ of which yielded malignant results (Table 4).

The median DI for the pandemic group was 1 day (range 158 days). For patients with a diagnosis not requiring tissue sampling, the median DI was 1 day (range 1-17 days). For patients with a diagnosis requiring tissue sampling, the median DI was 8 days (range, 1-58 days); those with a malignant result, 5 days (range 1-39 days); and those with a benign result, 9 days (range $1-58$ ).

\section{Diagnostic mammography pre-pandemic: April 2019- June 2019}

A total of 3920 patients underwent either screening or diagnostic mammography between April 1, 2019, and June 30, 2019. Sixty-four patients (cisgender female 62, cisgender male 2), with a median age of 57 years (range 30-85 years), underwent diagnostic mammography for either breast-related symptoms (40) or an abnormal surveillance mammogram (24). Breast-related symptoms/signs included palpable lump 
Table 2 Studies and procedures performed per visit during the COVID-19 pandemic, and one year prior during the pre-pandemic times

\begin{tabular}{|c|c|c|c|c|c|c|c|c|c|}
\hline \multirow[t]{2}{*}{ Imaging studies/biopsies } & \multicolumn{4}{|l|}{ Pandemic } & \multicolumn{5}{|c|}{ Pre-pandemic } \\
\hline & $\begin{array}{l}1^{\text {st }} \text { visit } \\
(n=145)\end{array}$ & $\begin{array}{l}2^{\text {nd }} \text { visit } \\
(n=52)\end{array}$ & $\begin{array}{l}3^{r d} \text { visit } \\
(n=11)\end{array}$ & $\begin{array}{l}4^{\text {th }} \text { visit } \\
(\mathrm{n}=1)\end{array}$ & $\begin{array}{l}1^{\text {st }} \text { visit } \\
(n=82)\end{array}$ & $\begin{array}{l}2^{\text {nd }} \text { visit } \\
(n=62)\end{array}$ & $\begin{array}{l}3^{\text {rd }} \text { visit } \\
(n=13)\end{array}$ & $\begin{array}{l}4^{\text {th }} \text { visit } \\
(n=4)\end{array}$ & $\begin{array}{l}\text { 5th visit } \\
(\mathrm{n}=2)\end{array}$ \\
\hline Bilateral mammogram & $19(13.1 \%)$ & 0 & 0 & 0 & $52(63.4 \%)$ & 0 & 0 & 0 & 0 \\
\hline Unilateral mammogram & $56(38.6 \%)$ & 0 & 0 & 0 & $10(12.2 \%)$ & 0 & 0 & 0 & 0 \\
\hline Compression views & $6(4.1 \%)$ & $13(25 \%)$ & 0 & 0 & $1(1.2 \%)$ & $19(30.6 \%)$ & $1(7.7 \%)$ & 0 & 0 \\
\hline Magnification views & $7(4.8 \%)$ & $6(11.5 \%)$ & 0 & 0 & $4(4.9 \%)$ & $10(16.1 \%)$ & 0 & 0 & 0 \\
\hline Bilateral ultrasound & $8(5.5 \%)$ & 0 & 0 & 0 & $8(9.8 \%)$ & 0 & 0 & 0 & 0 \\
\hline Unilateral ultrasound & $37(25.5 \%)$ & $12(23.1 \%)$ & $2(18.2 \%)$ & 0 & $3(3.7 \%)$ & $22(35.5 \%)$ & $6(46.2 \%)$ & 0 & 0 \\
\hline MRI & 0 & $3(5.8 \%)$ & $1(9.1 \%)$ & 0 & 0 & $4(6.5 \%)$ & $1(7.7 \%)$ & $2(50.0 \%)$ & 0 \\
\hline Stereotaxic-guided core biopsy & $1(0.7 \%)$ & $5(9.6 \%)$ & $3(27.3 \%)$ & 0 & $1(1.2 \%)$ & $4(6.5 \%)$ & $3(23.1 \%)$ & $1(25.0 \%)$ & 0 \\
\hline Ultrasound-guided core biopsy & $9(6.2 \%)$ & $11(21.2 \%)$ & $3(27.3 \%)$ & 0 & $3(3.7 \%)$ & $3(4.8 \%)$ & $2(15.4 \%)$ & $1(25.0 \%)$ & 0 \\
\hline Ultrasound-guided FNA & $2(3.8 \%)$ & $2(3.8 \%)$ & $2(18.2 \%)$ & 0 & 0 & 0 & 0 & 0 & 0 \\
\hline MRI-guided core biopsy & 0 & 0 & 0 & $1(100 \%)$ & 0 & 0 & 0 & 0 & $2(100 \%)$ \\
\hline
\end{tabular}

(30), nipple discharge (2), axillary fullness (2), pruritus (1), pain (4) and skin thickening (1).

At the initial visit, 64 patients underwent a total of 78 additional imaging studies and 4 core biopsies. Forty-nine patients returned for a second visit with a total of 55 imaging studies and 7 core biopsies. Twelve patients returned for a third visit, 7 patients for imaging and 5 for image-guided core biopsies. Four patients returned for a fourth visit, 2 patients for an MRI and 2 for core biopsies. Both patients who underwent MRI returned for the fifth visit for MRI-guided biopsies (Table 2).

\section{Diagnostic interval}

$23.4 \%$ of patients completed their assessment on the initial visit, $57.8 \%$ on the second visit, $12.5 \%$ on the third visit, $3.1 \%$ on the fourth visit and $3.1 \%$ on the fifth visit (Table 3). A total of 21 patients underwent core biopsy, 7 $(33.3 \%)$ of which yielded malignant results (Table 4$)$.
The median (DI) for the pre-pandemic group was 15 days (range 1-128 days). The median DI for patients with a diagnosis not requiring tissue sampling was 11 days (range 1-89 days). For patients with a diagnosis requiring tissue sampling, the median DI was 31 days (range 1-128 days); those with a malignant result, 9 days (range 1-37 days); and those with a benign result, 33 days (range 1-128).

\section{Comparison of diagnostic interval between COVID-19 pandemic (2020) and pre-pandemic (2019)}

During the pandemic period, the total number of patients presenting to the department was approximately seven times lower when compared to the same period one year prior, due to decreased screening. However, the percentage of patients referred for non-screening-related diagnostic mammography was higher during the pandemic (13.4\%) compared to the pre-pandemic period $(1.6 \%)(p<0.0001)$.

Table 3 Complete assessments per visit during the COVID-19 pandemic, and one year prior during the pre-pandemic times

\begin{tabular}{|c|c|c|c|c|c|c|}
\hline \multirow[t]{2}{*}{ Visit } & \multicolumn{3}{|l|}{$\begin{array}{l}\text { Pandemic } \\
\mathrm{n}=77\end{array}$} & \multicolumn{3}{|l|}{$\begin{array}{l}\text { Pre-pandemic } \\
n=64\end{array}$} \\
\hline & $\begin{array}{l}\text { Completed } \\
\text { assessments } \\
\text { No. }(\%)\end{array}$ & $\begin{array}{l}\text { Final benign } \\
\text { diagnosis } \\
\text { No. }(\%)\end{array}$ & $\begin{array}{l}\text { Final malignant } \\
\text { diagnosis } \\
\text { No. }(\%)\end{array}$ & $\begin{array}{l}\text { Completed } \\
\text { assessments } \\
\text { No. }(\%)\end{array}$ & $\begin{array}{l}\text { Final benign } \\
\text { diagnosis } \\
\text { No. }(\%)\end{array}$ & $\begin{array}{l}\text { Final malignant } \\
\text { diagnosis } \\
\text { No. }(\%)\end{array}$ \\
\hline 1 (initial) & $39(50.6 \%)$ & $33(42.9 \%)$ & $6(7.8 \%)$ & $15(23.4 \%)$ & $12(18.8 \%)$ & $3(4.7 \%)$ \\
\hline 2 & $30(39.0 \%)$ & $24(31.2 \%)$ & $6(7.8 \%)$ & $37(57.8 \%)$ & $34(53.1 \%)$ & $3(4.7 \%)$ \\
\hline 3 & $7(9.1 \%)$ & $4(5.2 \%)$ & $3(3.9 \%)$ & $8(12.5 \%)$ & $7(10.9 \%)$ & $1(1.6 \%)$ \\
\hline 4 & $1(1.3 \%)$ & 0 & $1(1.3 \%)$ & $2(3.1 \%)$ & $2(3.1 \%)$ & 0 \\
\hline 5 & N/A & N/A & N/A & $2(3.1 \%)$ & $2(3.1 \%)$ & 0 \\
\hline
\end{tabular}


Table 4 Pathology results from percutaneous core biopsy or FNA performed during the COVID-19 pandemic, and one year prior during the pre-pandemic times

\begin{tabular}{lll}
\hline BI-RADS classification & Pandemic $(\mathrm{n}=34)$ & Pre-pandemic $(\mathrm{n}=21)$ \\
\hline Ductal carcinoma in situ (DCIS) & $2(5.9 \%)$ & $1(4.8 \%)$ \\
Invasive ductal carcinoma (IDC) & $9(26.5 \%)$ & $5(23.8 \%)$ \\
IDC with axillary lymph node metastasis & $3(8.8 \%)$ & 0 \\
IDC and DCIS & $1(2.9 \%)$ & 0 \\
Invasive lobular carcinoma (ILC) & $1(2.9 \%)$ & 0 \\
Diffuse large B cell lymphoma & 0 & $1(4.8 \%)$ \\
Various benign pathologies & $16(47.1 \%)$ & $12(57.1 \%)$ \\
Atypical benign pathology results & $2(5.9 \%)$ & $2(9.5 \%)$ \\
\hline
\end{tabular}

When evaluated as a group, the patient cohort during the pandemic period showed a statistically significant reduction in the DI compared to the pre-pandemic group $(p<0.0001)$ (Fig. 2 ). In the pandemic group, a higher percentage of patients had their assessment completed on the initial visit (pandemic group 50.6\%; pre-pandemic group 23.4\%) ( $p=0.0009$ ), with fewer patients classified as BI-RADS 0 (27.3\%), compared to the pre-pandemic group $(64.1 \%)(p=0.0001)$ (Table 5). A final diagnosis was made for most patients (percentile $90^{\text {th }}$ ) within 21 days during the pandemic compared to 63 days in the pre-pandemic times (Fig. 2).

In the pandemic group, there was a significant reduction in the DI for patients not requiring tissue sampling for diagnosis $\left(p<0.0001 ; 90^{\text {th }}\right.$ percentile 8 days in 2020 and 55 days in 2019), as well as for patients requiring tissue sampling for diagnosis $\left(p=0.0028 ; 90^{\text {th }}\right.$ percentile 28 days in 2020 and 91 days in 2019), compared to the pre-pandemic group (Fig. $3)$. However, while patients in the pandemic group with a final benign diagnosis showed a reduction in DI $\left(p=0.0001 ; 90^{\text {th }}\right.$ percentile 28 days in 2020 and 126 days in 2019), those with a final malignant diagnosis showed no significant difference in DI ( $p=0.9177 ; 90^{\text {th }}$ percentile 37 days in 2020 and 37 days in 2019) when compared to the pre-pandemic group (Fig. 4).
There was no statistically significant difference in the percentage of patients undergoing percutaneous breast biopsies in the pandemic group compared to the pre-pandemic group (pandemic group 44.2\%; pre-pandemic group 32.8\%) ( $p=$ 0.1692 ). There was also no difference in the percentage of biopsies showing malignancy during the pandemic period (47.1\%) compared to the pre-pandemic time $(33.3 \%)(p=$ $0.3161)$.

\section{Discussion}

Our study showed a statistically significant decrease in the diagnostic interval for patients from the pandemic group who did not require tissue sampling for completion of their assessment, as well as for patients requiring tissue sampling with a final benign diagnosis. In addition, more patients had their assessment completed at a single initial visit during the pandemic compared to the pre-pandemic group.

The restructuring of the breast imaging department to a Rapid Diagnostic Unit-type practice that occurred as a result of the pandemic could account for the observed reduction in diagnostic
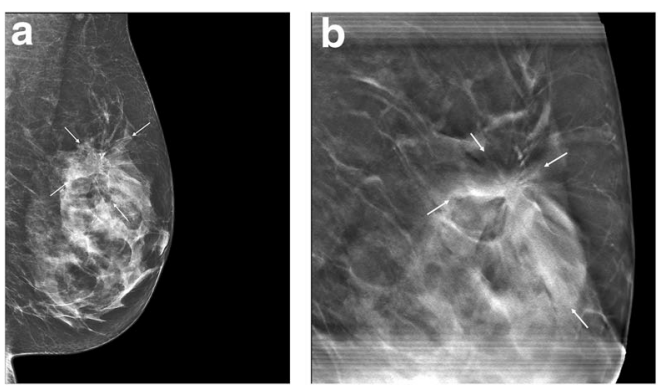

Fig. 1 Fifty-four-year-old woman presented with palpable regions in both breasts. All imaging tests and biopsies were completed on a single visit. Left breast: a left breast mediolateral oblique projection from the initial mammogram shows architectural distortion in the upper aspect of that breast (arrows). b Spot tomosynthesis view of the upper-left breast shows persistence of the architectural distortion (arrows). $\mathbf{c}$ On left breast ultrasound, the architectural distortion corresponds to a 1.9-cm solid mass (arrows). Right breast: $\mathbf{d}$ right breast craniocaudal projection from the
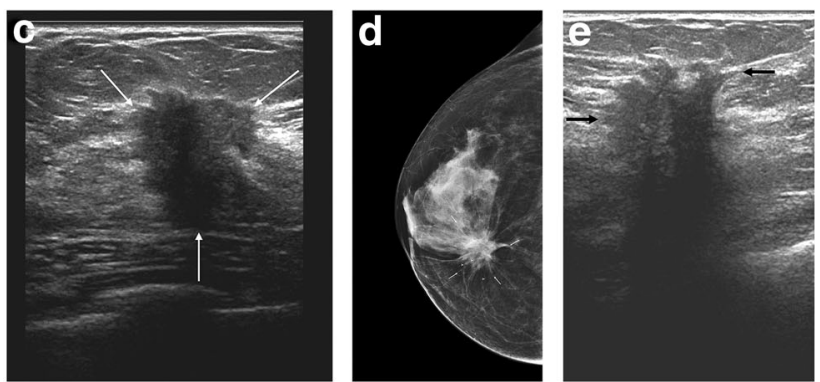

initial mammogram shows a spiculated mass (arrows) associated with pleomorphic calcifications (arrowheads) in the medial right breast. e On right breast ultrasound, this corresponds to a $2.9-\mathrm{cm}$ solid mass (arrows). Bilateral ultrasound core biopsies showed bilateral invasive ductal carcinomas. The pathology report was available 4 days later at which time the patient was contacted by a breast surgeon to discuss a treatment plan. The surgery was performed 10 days after the pathology results were available 
Table 5 BI-RADS classification after the initial visit during the COVID-19 pandemic, and one year prior during the pre-pandemic time

\begin{tabular}{lll}
\hline BI-RADS classification & Pandemic $(\mathrm{n}=77)$ & Pre-pandemic $(\mathrm{n}=64)$ \\
\hline BI-RADS 1 & $5(6.5 \%)$ & $2(3.1 \%)$ \\
BI-RADS 2 & $19(24.7 \%)$ & $8(12.5 \%)$ \\
BI-RADS 3 & $4(5.2 \%)$ & $1(1.6 \%)$ \\
BI-RADS 4 & $24(31.2 \%)$ & $10(15.6 \%)$ \\
BI-RADS 5 & $4(5.2 \%)$ & $2(3.1 \%)$ \\
BI-RADS 0 & $21(27.3 \%)$ & $41(64.1 \%)$ \\
\hline
\end{tabular}

intervals. Patients who required diagnostic mammography were guided through the assessment process by a navigator team, which expanded from pre-pandemic times to now include technologists, radiologists, surgeons, secretaries and reception staff. There was also a shift in the milieu of the department during the pandemic, where all members of the breast imaging group were motivated to complete the assessment of each patient on the initial visit, and if not possible, with a minimal number of visits. This permitted increased flexibility in the booking schedule as the healthcare team would willingly adjust workflow on short notice to facilitate expedited diagnostic care. Despite this flexibility, some patients returned for follow-up tests due to personal scheduling conflicts or booking challenges securing same-day procedures such as MRI studies, which would account for the observed range of DI values. Nevertheless, the combination of an expanded navigation team coupled with a highly motivated department was paramount in expediting care in this patient-centred model.

Rapid Diagnostic Units have been shown to substantially reduce wait times to definitive diagnosis, with improved satisfaction scores among patients $[16,17]$. Racz et al showed a

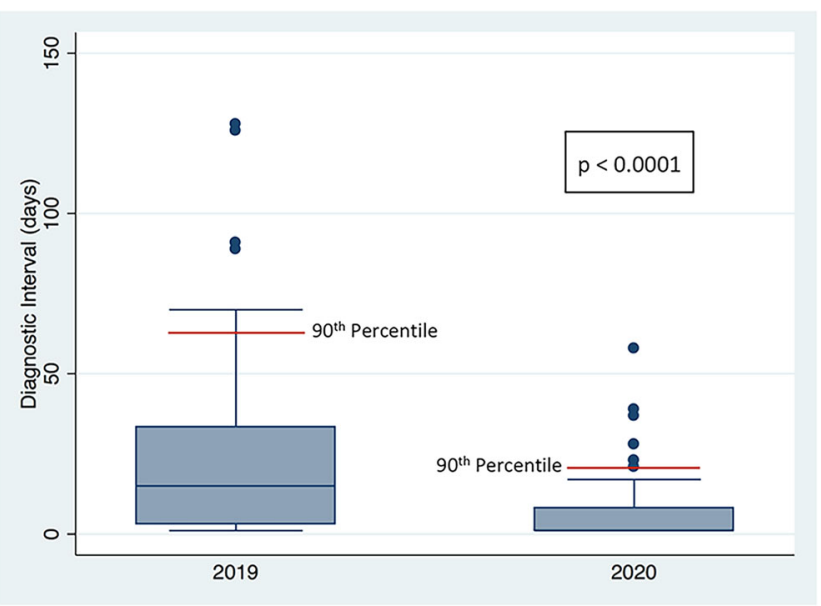

Fig. 2 Box plots showing the diagnostic interval (DI) in days during the 2020 pandemic and the 2019 pre-pandemic times. There is a statistically significant difference in the DI between 2020 and $2019(p<0.0001)$. A final diagnosis was made for most of the patients (percentile $90^{\text {th }}$; red line) in 21 days during the pandemic as compared to 63 days in the prepandemic times

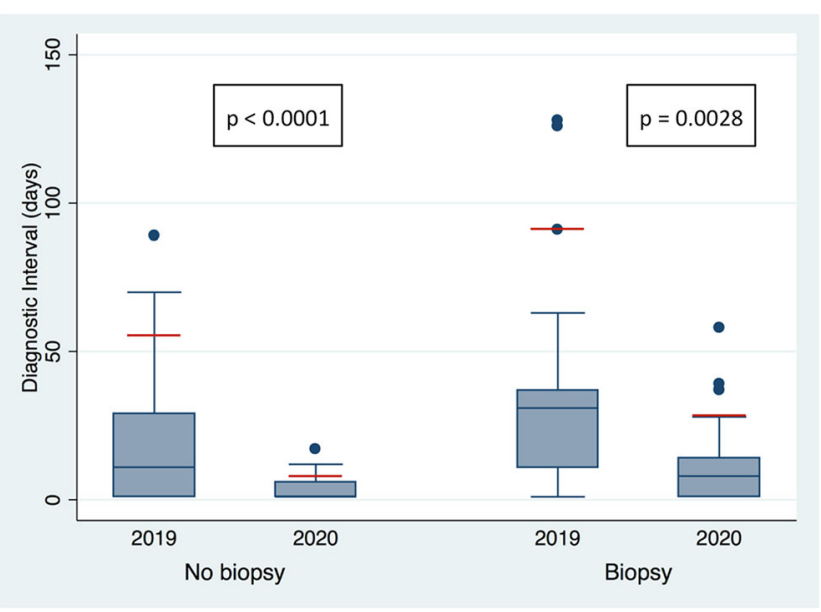

Fig. 3 Box plots showing the diagnostic interval (DI) in days during the 2020 pandemic and the 2019 pre-pandemic times among patients who did and did not undergo breast biopsy. Statistically significant differences were found in DI in both groups (biopsy group $p=0.0028$, no biopsy group $p<0.0001)$. In the pandemic group, a final diagnosis was made for most of the patients $\left(90^{\text {th }}\right.$ percentile; red line) requiring biopsy in 28 days and not requiring biopsy in 8 days, as compared with 91 days and 55 days, respectively, in the pre-pandemic group

significant reduction in wait times from initial consultation to diagnosis and a greater chance of receiving neoadjuvant chemotherapy in 260 patients in a rapid diagnostic unit compared to 287 patients in a non-rapid diagnostic clinic [17]. Arnaout et al evaluated 211 patients in a Rapid Diagnosis and Support Clinic and showed an improvement in diagnostic wait times, satisfaction scores and understanding of treatment plans in

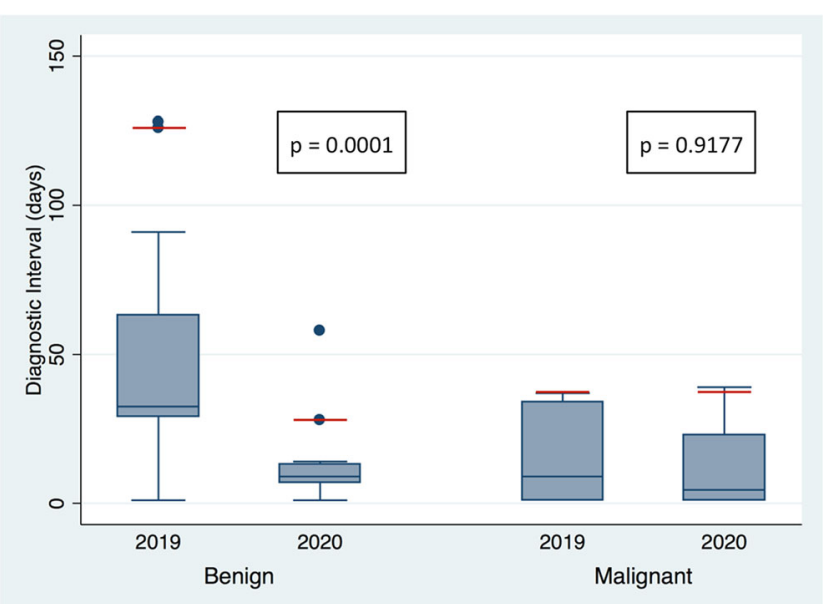

Fig. 4 Box plots showing the diagnostic interval (DI) in days during the 2020 pandemic and the 2019 pre-pandemic times among patients who had benign and malignant biopsy pathology results. A statistically significant difference was found in DI among patients with a benign diagnosis $(p=0.0001)$. No statistically significant difference was found in the group of patients with a malignant diagnosis $(p=0.9177)$. In the pandemic group, a final diagnosis was made for most of the patients $\left(90^{\text {th }}\right.$ percentile; red line) with a benign result in 28 days and a malignant result in 37 days, as compared with 126 days and 37 days, respectively, in the prepandemic group 
patients with initial breast imaging tests classified as BIRADS 5 [16].

Our study showed that there was no change in the diagnostic interval for patients requiring biopsy with a final malignant diagnosis compared to pre-pandemic times. This can be explained by prioritisation of worrisome cases, where there is a high suspicion of breast cancer by the physician, and would appear to occur irrespective of whether the evaluation occurs during a pandemic or non-pandemic times. The concept of expedited assessments associated with a decreased likelihood of delays in breast cancer diagnosis has been previously described in patients who present with highly suspicious mammograms, large tumours and advanced breast cancer stages [14, 18, 19]. Although there was no change in diagnostic interval in these patients, the present care model would suggest that the treatment time may be reduced since patients that underwent a biopsy were contacted by a breast surgeon within $24 \mathrm{~h}$ of the pathology report becoming available to discuss a management plan.

The COVID-19 pandemic has had a major impact on the mental health of many societies, with symptoms including anxiety, mood swings, depression, insomnia, irritability and anger. The prevalence of COVID-19-related anxiety and depression has also been shown to be higher in women when compared to men [12]. Anxiety related to the fear of acquiring COVID-19 has also been suggested as a possible cause for women with breast cancer or a suspicious breast lesion in need of assessment, declining procedures or surgeries during the pandemic [20]. Rapid assessment processes in breast imaging, with a reduction in time to diagnosis, have been associated with a decrease in patient anxiety and physiological distress, most notably in the short term [17, 19]. Therefore, during a pandemic, reducing the diagnostic interval in patients with a high clinical suspicion of breast cancer would be of substantial benefit to their mental health and may reduce patient-directed treatment delays.

Providing a breast imaging service that focuses on reducing diagnostic wait times requires a continuous on-site physician presence. The need for breast imaging physicians to be physically present at the facility may diverge from the scheduling structure of the remaining radiology department, in that, during the pandemic, there was a transition from on-site reporting to incorporating off-site reporting, with some practices permitting up to $80 \%$ of staff to report from home workstations [21]. Despite this trend, it is recognised that off-site reporting occurs less frequently in breast imaging sections due to the challenges of setting up high-resolution remote reporting systems, which could come at a considerable expense, and the potential requirement of physicians to directly manage complex diagnostic cases [22]. In order to alter the diagnostic interval during the pandemic, breast imagers should anticipate a higher proportion of on premise work compared to their colleagues, which comes with a potential higher risk of viral exposure.

There are limitations to this single-site study. There may have been a selection bias as only patients with a high clinical suspicion of breast cancer, who were asymptomatic for COVID-19, were included in the pandemic group. This was unavoidable as facility guidelines required limitations on outpatient services and a stringent triage process in order to prevent potential viral exposure, resulting in a small study population. Nevertheless, we believe that our results are generalizable to breast centres during a pandemic that faces similar restrictions on patient numbers and policies set to protect patient and healthcare worker exposure. Despite the small study population, the reduction in the diagnostic interval in the pandemic group is consistent with the decrease in wait times observed in rapid diagnostic models in the literature and supports the benefit of adopting such a model even during non-pandemic times [16, 17]. However, restructuring permanently to such a model would entail coordinated patient navigation, a flexible booking platform, continuous radiologist presence and cooperation from non-radiology services as breast surgery.

The challenges of the pandemic motivated our department to reassess our operational efficiencies and restructure to a Rapid Diagnostic Unit with a reduction in DI in patients with a high clinical suspicion for breast cancer. The benefits gained from implementing such a patient-centric model in these unprecedented times would appear to justify its adoption in breast imaging departments once the pandemic has ended. Furthermore, the concept of expedited imaging follow-up to final diagnosis, guided by a team of navigators and overseen by a group of healthcare professionals intent on a rapid turnaround, can potentially be extended beyond breast imaging to other areas of the radiology where a multimodality approach to diagnosis is utilised.

Funding The authors state that this work has not received any funding.

\section{Declarations}

Guarantor The scientific guarantor of this publication is Derek Muradali.

Conflict of interest The authors of this manuscript declare no relationships with any companies whose products or services may be related to the subject matter of the article.

Statistics and biometry One of the authors has significant statistical expertise.

Informed consent Written informed consent was waived by the Institutional Review Board.

Ethical approval Institutional Review Board approval was obtained.

\section{Methodology}

- Retrospective

- Observational

- Performed at one institution 


\section{References}

1. Seely JM, Scaranelo AM, Yong-Hing C et al (2020) COVID-19: safe guidelines for breast imaging during the pandemic. Can Assoc Radiol J 71:459-469

2. Dinmohamed AG, Visser O, Verhoeven RHA et al (2020) Fewer cancer diagnoses during the COVID-19 epidemic in the Netherlands. Lancet Oncol 21:750-751

3. Gathani T, Clayton G, MacInnes E, Horgan K (2021) The COVID19 pandemic and impact on breast cancer diagnoses: what happened in England in the first half of 2020. Br J Cancer 124:710 712. https://doi.org/10.1038/s41416-020-01182-z

4. Feletto E, Grogan P, Nickson C et al (2020) How has COVID-19 impacted cancer screening? Adaptation of services and the future outlook in Australia. Public Health Res Pract 30(4):3042026. https://doi.org/10.17061/phrp3042026

5. McKibbin, Warwick J. and Fernando, Roshen, The Global Macroeconomic Impacts of COVID-19: Seven Scenarios (March 2, 2020). CAMA Working Paper No. 19/2020, Available at https:// doi.org/10.2139/ssrn.3547729

6. Curigliano G, Cardoso MJ, Poortmans P et al (2020) Recommendations for triage, prioritisation and treatment of breast cancer patients during the COVID-19 pandemic. Breast 52:8-16. https://doi.org/10.1016/j.breast.2020.04.006

7. Spring LM, Specht MC, Jimenez RB et al (2020) Case 22-2020: a 62-year-old woman with early breast cancer during the Covid-19 pandemic. N Engl J Med 383:262-272. https://doi.org/10.1056/ nejmcpc2002422

8. Olivotto IA, Gomi A, Bancej C et al (2002) Influence of delay to diagnosis on prognostic indicators of screen-detected breast carcinoma. Cancer 94:2143-2150. https://doi.org/10.1002/cncr.10453

9. Vujovic O, Yu E, Cherian A et al (2009) Effect of interval to definitive breast surgery on clinical presentation and survival in early-stage invasive breast cancer. Int J Radiat Oncol Biol Phys 75:771-774. https://doi.org/10.1016/j.ijrobp.2008.11.049

10. Chen Z, King W, Pearcey R et al (2008) The relationship between waiting time for radiotherapy and clinical outcomes: a systematic review of the literature. Radiother Oncol 87:3-16

11. Huang J, Barbera L, Brouwers M et al (2003) Does delay in starting treatment affect the outcomes of radiotherapy? A systematic review. J Clin Oncol 21:555-563. https://doi.org/10.1200/JCO. 2003.04.171

12. Salari N, Hosseinian-Far A, Jalali R, et al. (2020) Prevalence of stress, anxiety, depression among the general population during the COVID-19 pandemic: a systematic review and meta-analysis.
Global Health. 16(1):57. https://doi.org/10.1186/s12992-02000589-w

13. D'Orsi CJ SEME et al (2013) ACR BI-RADS ${ }^{\circledR}$ Atlas, Breast Imaging Reporting and Data System. Reston, VA, American College of Radiology

14. Chiarelli AM, Muradali D, Blackmore KM et al (2017) Evaluating wait times from screening to breast cancer diagnosis among women undergoing organised assessment vs usual care. Br J Cancer 116: 1254-1263. https://doi.org/10.1038/bjc.2017.87

15. Groome PA, Webber C, Whitehead M et al (2019) Determining the cancer diagnostic interval using administrative health care data in a breast cancer cohort. JCO Clin Cancer Inform 3:1-10. https://doi. org $/ 10.1200 /$ CCI.18.00131

16. Arnaout A, Smylie J, Seely J et al (2013) Improving breast diagnostic services with a rapid access diagnostic and support (RADS) program. Ann Surg Oncol 20:3335-3340. https://doi.org/10.1245/ s10434-013-3120-5

17. Racz JM, Holloway CMB, Huang W, Look Hong NJ (2016) Improving patient flow and timeliness in the diagnosis and management of breast abnormalities: the impact of a rapid diagnostic unit. Curr Oncol 23:e260-e265. https://doi.org/10.3747/co.23. 3017

18. Jiang L, Gilbert J, Langley H et al (2015) Effect of specialized diagnostic assessment units on the time to diagnosis in screen-detected breast cancer patients. Br J Cancer 112:1744-1750. https:// doi.org/10.1038/bjc.2015.147

19. Caplan LS, May DS, Richardson LC et al (2000) Time to diagnosis and treatment of breast cancer: results from the National Breast and Cervical Cancer Early Detection Program, 1991-1995. Am J Public Health 90(1):130-134. https://doi.org/10.2105/ajph.90.1.130

20. Vanni G, Materazzo M, Pellicciaro M et al (2020) Breast cancer and COVID - 19: the effect of fear on patients' decision-making process. In Vivo 34:1651-1659. https://doi.org/10.21873/invivo. 11957

21. Sammer MBK, Sher AC, Huisman TAGM, Seghers VJ (2020) Response to the COVID-19 pandemic: practical guide to rapidly deploying home workstations to guarantee radiology services during quarantine, social distancing, and stay home orders. AJR Am J Roentgenol 215:1417-1420

22. Freer PE (2021) The impact of the COVID-19 pandemic on breast imaging. Radiol Clin North Am 59:1-11

Publisher's note Springer Nature remains neutral with regard to jurisdictional claims in published maps and institutional affiliations. 